\title{
Fungos conidiais em Euterpe oleracea Mart. (açaizeiro) na Ilha do Combu, Pará-Brasil
}

\author{
Carla Corrêa de Castro ${ }^{1}$, Antonio Hernández Gutiérrez ${ }^{2}$ e Helen Maria Pontes Sotão ${ }^{1,3}$
}

Recebido em 12/04/2011. Aceito em 18/06/2012

\begin{abstract}
RESUMO
(Fungos Conidiais em Euterpe oleracea Mart. (açaizeiro) na Ilha do Combu, Pará-Brasil). O trabalho teve como objetivo investigar a ocorrência de fungos conidiais (hyphomycetes) associados à decomposição de material vegetal morto de Euterpe oleracea Mart. (açaizeiro) na Área de Proteção Ambiental da ilha do Combu, município de Belém, Pará. Entre agosto de 2008 e abril de 2009, foram coletadas partes em decomposição de palmeiras de E. oleracea e mantidas em câmara-úmida durante sete dias. As estruturas reprodutivas dos fungos foram retiradas e montadas em lâminas semipermanentes para o estudo morfológico em microscopia óptica. O presente trabalho contém descrições, comentários, distribuição geográfica e ilustrações dos novos registros para o Brasil. Como resultados deste estudo foram identificados 45 táxons de hifomicetos. Todos são novos registros para a área de estudo, destes 11 representam novos registro para o Brasil e 12 para a Amazônia brasileira.
\end{abstract}

Palavras-chave: açai, Amazônia, hyphomycetes, palmeira

\begin{abstract}
(Conidial Fungi on Euterpe oleracea Mart. on Combu Island, Pará, Brazil). The objective of this study was look for conidial fungi (hyphomycetes) associated with decomposing, dead vegetative material of Euterpe oleracea Mart. collected on Combu Island (Belém, Pará), an environmentally protected area. From August 2008 to April 2009, decomposing parts of E. oleracea were collected and kept in a moist chamber for seven days. Somatic and reproductive fungal structures were extracted from the substrate and mounted on semi-permanent slides for morphological study using optical microscopy. This paper includes descriptions, comments, geographical distributions and illustrations of new records for Brazil. All these records are new for the study area, 11 are new records for Brazil, and 12 are new for the Brazilian Amazon.
\end{abstract}

Key words: açaí, Amazonian, hyphomycetes, palm

\section{Introdução}

$\mathrm{O}$ açaizeiro (Euterpe oleracea Mart.; Arecaceae Bercht. \& J. Presl) é uma palmeira de grande interesse econômico na região amazônica; os seus frutos assim como o palmito dele extraído são uns dos principais recursos na sua comercialização (Pimentel \& Jardim, 2009). Ocorre no estuário amazônico, encontrado principalmente nas matas de várzea e igapó do baixo Amazonas, Tocantins e Maranhão, assim como em Amapá, Guianas e Venezuela (Silva et al. 2005a).

Os hifomicetos constituem uma classe artificial de fungos, incluída no grupo dos fungos conidiais (anamorfos). Estes fungos se reproduzem assexuadamente através da pro- dução de conídios, formados por estruturas especializadas denominadas conidióforos, originadas a partir das hifas. Os conídios têm como principais funções a dispersão e a perpetuação da espécie, apresentando grande importância ecológica e taxonômica (Barbosa et al. 2008). Muitas espécies de hifomicetos causam doenças em diversas plantas de interesse econômico (Silva et al. 2005b; Furtado et al. 2007).

A ilha do Combu é uma Área de Proteção Ambiental (APA), localizada na região metropolitana de Belém, Estado do Pará. Apresenta uma vegetação com estrutura e composição florística variada, incluindo floresta primária e secundária, onde o açaizeiro é a espécie silvestre mais abundante e de maior importância econômica (Rodrigues et al. 2006).

\footnotetext{
${ }^{1}$ Museu Paraense Emílio Goeldi, Coordenação de Botânica, Laboratório de Micologia, Belém, PA, Brasil

${ }^{2}$ Universidade Federal do Pará, Instituto de Ciências Biológicas, Belém, PA, Brasil

${ }^{3}$ Autor para correspondência: helen@museu-goeldi.br
} 
De acordo com Hyde et al. (1997), aproximadamente 1.580 fungos foram registrados sobre palmeiras, incluindo 660 fungos anamorfos, o que indica que esses vegetais suportam alta diversidade de fungos. Na Amazônia brasileira, são poucos os estudos envolvendo fungos sobre palmeiras (Farr 1980; Rodrigues \& Samuels 1992; Hernández et al. 2009; Monteiro et al. 2010). Os trabalhos de Rodrigues (1994) e Negrão et al. (2009) relacionaram os primeiros registros de hifomicetos associados a palmeiras do açaizeiro (E. oleraceae) para a ilha do Combu, mais recentemente, fazendo parte do levantamento realizado neste trabalho Castro et. al. (2011) publicaram dois novos registros para o neotrópico e dez para América do Sul.

Considerando a carência de levantamentos sobre a diversidade dos fungos e o acelerado desmatamento da Floresta Amazônica, a informação obtida neste estudo é relevante para o conhecimento da distribuição e diversidade destes organismos. O objetivo deste estudo foi investigar a ocorrência de fungos conidiais (hifomicetos) associados à decomposição de partes mortas do açaizeiro (E. oleracea) na APA da ilha do Combu.

\section{Material e métodos}

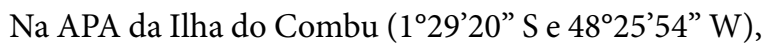
localizada no município de Belém, Pará, foram realizadas coletas de partes em decomposição de E. oleracea, entre agosto de 2008 e abril de 2009. As coletas foram efetuadas em oito transectos de $100 \times 10 \mathrm{~m}$, em vegetação do tipo floresta de várzea, com cinco amostras por transecto, sendo a disponibilidade de material em decomposição o critério utilizado para a escolha da planta a ser coletada a cada intervalo de $20 \mathrm{~m}$.

O método de coleta foi baseado na busca de partes em decomposição da palmeira (bainhas, cachos, espatas, estipes, folíolos, pecíolos, raízes e raques). O material coletado foi mantido em câmara-úmida por sete dias; após esse período, foi colocado para secar em estufa elétrica a uma temperatura de aproximadamente $60^{\circ} \mathrm{C}$, por 24 horas e, finalmente, mantido sob refrigeração $\left(-18^{\circ} \mathrm{C}\right)$ para evitar a invasão e deterioração por ácaros.

O exame das amostras seguiu a metodologia convencional adotada para o grupo, conforme descrita por Monteiro et al. (2010). As lâminas foram observadas ao microscópio óptico com luz clara, para análise das microestruturas.

As amostras estudadas foram depositadas no Herbário João Murça Pires (MG), do Museu Paraense Emílio Goeldi.

Neste trabalho são apresentadas descrições, comentários taxonômicos, distribuição geográfica e ilustrações para as espécies que representam primeiros registros no Brasil.

\section{Resultados e discussão}

Foram registrados 45 táxons de fungos conidiais colonizando partes em decomposição de E. oleracea. Todas as espécies identificadas são novos registros para a área de estudo, destes 11 representam novos registros para o Brasil e 12 para a Amazônia brasileira. (Tab. 1).

A maioria dos táxons foram coletados sobre bainhas (26), seguido de cachos (20) e cascas (20). Os substratos com menor número de táxons encontrados foram estipes (3) e raízes (3). Seis espécies ocorreram exclusivamente em raques e somente cinco em bainhas. Cachos, espatas e raízes apresentaram duas espécies exclusivas cada um, enquanto folíolos e pecíolos apenas uma espécie (Tab. 1).

Os gêneros Chalara (Corda) Rabenh., Curvularia Boedijn e Cylindrocladium Morgan são citados na literatura causando patogenicidade em plantas de interesse econômico como Daucus carotae L. (cenoura), Zoysia japonica Steud. (gramínea) e Roystonea sp. (palmeira ornamental), respectivamente (Dalbosco et al. 2004; Nechet \& Halfeld-Vieira 2005; Russomanno et al. 2007).

A expressiva constatação de uma diversificada micobiota de fungos conidiais associados a plantas de E. oleraceae (açaí), assim como o achado de vários primeiros registros contribuem significativamente para a ampliação do conhecimento da diversidade desses fungos no bioma Amazônia e servem como indicativo para subsidiar estudos mais aprofundados relacionados às relações ecológicas com este substrato.

Novos Registros para o Brasil:

1. Acarocybellina arengae (Matsush.) Subram., Proc. Indian natn Sci. Acad., Part B. Biol. Sci. 58(4): 187. 1992.

$\equiv$ Sporidesmium arengae Matsush., Icon. microfung. Matsush. lect. (Kobe): 136. 1975.

Fig. 1A

Colônias efusas em substrato natural. Conidióforos macronemáticos, mononemáticos, solitários, cilíndricos, eretos, retos, septados, ramificados no ápice, com 1-2 proliferações em forma de Y, lisos, castanho-avermelhados, 40-133 ×5-8 $\mu \mathrm{m}$, na célula basal das proliferações se produz uma hifa lateral que se curva para baixo, de cor castanha na base, clareando em direção à extremidade livre, muitas vezes aderidas ao conidióforo. Células conidiogênicas monotréticas, terminais, discretas, percorrentes, cilíndricas, lisas a raramente verrucosas, castanhas. Conídios solitários, 3-septados, obovoides, simples, lisos, secos, uniformemente castanhos ou com a célula basal mais clara, 24-33 × 11-15 $\mu \mathrm{m}$, base truncada com $3 \mu \mathrm{m}$ de largura.

Material examinado: BRASIL. Pará: Belém, Ilha do Combu, 20/VIII/2008, sobre bainhas, C.C. Castro 54 (MG195841); ibid, 18/III/2009, sobre espatas, C.C. Castro 75 (MG199676).

Comentários: Acarocybellina é um gênero monotípico estabelecido por Subramanian (1992) para acomodar Sporidesmium Arengae Matsuh. Segundo Hughes (1978) Acarocybellina se diferencia dos demais gêneros de hifomicetos por apresentar conidióforos com sucessivas proliferações em forma de $\mathrm{V}$ e pela produção de uma hifa lateral recurvada para baixo que cresce na base da proliferação. No entanto, Rao et al. (1981) estabeleceram o gênero monotípico 
Tabela 1. Fungos conidiais encontrados em açaizeiro na APA da Ilha do Combu. Partes do açaizeiro: $\mathrm{Ba}=$ Bainhas, $\mathrm{Ca}=\mathrm{Cachos}, \mathrm{Ep}=\mathrm{Espatas}$, Et $=\mathrm{Estipes}$, Fo $=$ Folíolos, $\mathrm{Pe}=$ Pecíolos, $\mathrm{Rz}=$ Raizes, $\mathrm{Rq}=$ Raques. Novos registros: $\left(^{*}\right)$ Amazônia brasileira, $\left.{ }^{*}\right)$ Brasil .

\begin{tabular}{|c|c|c|c|c|c|c|c|c|}
\hline \multirow{2}{*}{ Fungos conidiais } & \multicolumn{8}{|c|}{ Partes do açaizeiro } \\
\hline & $\mathrm{Ba}$ & $\mathrm{Ca}$ & Ep & Et & Fo & $\mathrm{Pe}$ & $\mathrm{Rz}$ & $\mathrm{Rq}$ \\
\hline${ }^{* *}$ Acarocybellina arengae (Matsush.) Subram. & $\mathrm{x}$ & & $\mathrm{x}$ & $\mathrm{x}$ & & & & $\mathrm{x}$ \\
\hline${ }^{*}$ Acrogenospora sphaerocephala (Berk. \& Broome) M.B. Ellis & & & & & & & $\mathrm{x}$ & \\
\hline Beltrania rhombica Penz. & & & & & $\mathrm{x}$ & & & \\
\hline${ }^{*}$ Beltraniella portoricensis (F. Stevens) Piroz. \& S.D. Patil & $\mathrm{x}$ & & & & $\mathrm{x}$ & & & \\
\hline Brachysporiella gayana Bat. & $\mathrm{x}$ & & & & & & & \\
\hline${ }^{*}$ Canalisporium caribense (Hol.-Jech. \& Mercado) Nawawi \& Kuthub. & $\mathrm{x}$ & $\mathrm{x}$ & & $\mathrm{x}$ & & & $\mathrm{x}$ & \\
\hline Chalara sp. & $\mathrm{x}$ & $\mathrm{x}$ & & & & & & \\
\hline Circinotrichum olivaceum (Speg.) Piroz. & & $\mathrm{x}$ & & & & & & $\mathrm{x}$ \\
\hline${ }^{* *}$ Coleodictyospora micronesiaca (Matsush.) Matsush. & $\mathrm{x}$ & $\mathrm{x}$ & $\mathrm{x}$ & & & $\mathrm{x}$ & & $\mathrm{x}$ \\
\hline${ }^{* *}$ Corynesporopsis inaequiseptata Matsush. & & & & & & & & $\mathrm{x}$ \\
\hline${ }^{* *}$ Corynesporopsis isabelicae Hol.-Jech. & $\mathrm{x}$ & $\mathrm{x}$ & & & & $\mathrm{x}$ & & $\mathrm{x}$ \\
\hline${ }^{*}$ Cryptophiale udagawae Piroz. \& Ichinoe & $\mathrm{x}$ & & & & & & & \\
\hline Curvularia lunata (Wakker) Boedijn & & & $\mathrm{x}$ & & & & & \\
\hline Cylindrocladium sp. & $\mathrm{x}$ & & & & $\mathrm{x}$ & & & $\mathrm{x}$ \\
\hline Dictyochaeta assamica (Agnihothr.) Aramb. & & $\mathrm{x}$ & & & & $\mathrm{x}$ & & \\
\hline${ }^{*}$ Dictyochaeta fertilis (S. Hughes \& W.B. Kendr.) Hol.-Jech. & & $\mathrm{x}$ & & & & $\mathrm{x}$ & & \\
\hline${ }^{*}$ Dictyochaeta simplex (S. Hughes \& W.B. Kendr.) Hol.-Jech. & $\mathrm{x}$ & & & & & & & \\
\hline Ellisembia adscendens (Berk.) Subram. & $\mathrm{x}$ & $\mathrm{x}$ & & & $\mathrm{x}$ & & & \\
\hline${ }^{\star}$ Ellisembia brachypus (Ellis \& Everh.) Subram. & $\mathrm{x}$ & & & & $\mathrm{x}$ & & & \\
\hline Ellisembia leonensis (M.B. Ellis) McKenzie & $\mathrm{x}$ & & & & & & & \\
\hline Endocalyx melanoxanthus (Berk. \& Broome) Petch & $\mathrm{x}$ & $\mathrm{x}$ & $\mathrm{x}$ & & & & & \\
\hline Exserticlava triseptata (Matsush.) S. Hughes & & & $\mathrm{x}$ & & & & & $\mathrm{x}$ \\
\hline Exserticlava vasiformis (Matsush.) S. Hughes & $\mathrm{x}$ & $\mathrm{x}$ & & & & $\mathrm{x}$ & & $\mathrm{x}$ \\
\hline 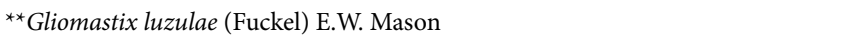 & & & & & & & & $\mathrm{x}$ \\
\hline${ }^{*}$ Gyrothrix podosperma (Corda) Rabenh. & $\mathrm{x}$ & & & & & & & \\
\hline ** Helicoma dennisii M.B. Ellis & & & & & & & & $\mathrm{x}$ \\
\hline${ }^{* *}$ Helicoma viridis (Corda) S. Hughes & & & & & & $\mathrm{x}$ & & \\
\hline${ }^{* *}$ Helminthosporium palmigenum Matsush. & & $\mathrm{x}$ & & & & & & \\
\hline${ }^{*}$ Melanographium citri (Gonz. Frag. \& Cif.) M.B. Ellis & & $\mathrm{x}$ & & & & $\mathrm{x}$ & & $\mathrm{x}$ \\
\hline${ }^{\star}$ Phialocephala humicola S.C. Jong \& E.E. Davis & & $\mathrm{x}$ & & & & & & \\
\hline Physalidiella elegans (Mosca) Rulamort & $\mathrm{x}$ & $\mathrm{x}$ & & & & & & $\mathrm{x}$ \\
\hline Piricauda cochinensis (Subram.) M.B. Ellis & $\mathrm{x}$ & $\mathrm{x}$ & $\mathrm{x}$ & & & $\mathrm{x}$ & & $\mathrm{x}$ \\
\hline${ }^{* *}$ Repetophragma moniliforme (Matsush.) R.F. Castañeda, McKenzie \& K.D. Hyde & & & & & & & & $\mathrm{x}$ \\
\hline **Spadicoides macrocontinua Matsush. & & & & & & & & $\mathrm{x}$ \\
\hline Spegazzinia sp. & & & $\mathrm{x}$ & & & & & \\
\hline Sporidesmium ghanaense M.B. Ellis & $\mathrm{x}$ & $\mathrm{x}$ & & & $\mathrm{x}$ & $\mathrm{x}$ & & $\mathrm{x}$ \\
\hline Sporidesmium macrurum (Sacc.) M.B. Ellis & $\mathrm{x}$ & $\mathrm{x}$ & $\mathrm{x}$ & $\mathrm{x}$ & & & & \\
\hline Sporidesmium tropicale M.B. Ellis & & & & & & & $\mathrm{x}$ & \\
\hline Sporoschisma saccardoi E.W. Mason \& S. Hughes & $\mathrm{x}$ & $\mathrm{x}$ & & & & & & \\
\hline Stachybotrys bisbyi (Sriniv.) G.L. Barron & $\mathrm{x}$ & $\mathrm{x}$ & & & $\mathrm{x}$ & $\mathrm{x}$ & & $\mathrm{x}$ \\
\hline Tetraploa aristata Berk. \& Broome & $\mathrm{x}$ & $\mathrm{x}$ & $\mathrm{x}$ & & & $\mathrm{x}$ & & $\mathrm{x}$ \\
\hline *Trichocladium opacum (Corda) S. Hughes & & & & & & & & $\mathrm{x}$ \\
\hline Zygosporium echinosporum Bunting \& E.W. Mason & $\mathrm{x}$ & & $\mathrm{x}$ & & $\mathrm{x}$ & $\mathrm{x}$ & & \\
\hline Zygosporium gibbum (Sacc., M. Rousseau \& E. Bommer) S. Hughes & $\mathrm{x}$ & & $\mathrm{x}$ & & $\mathrm{x}$ & & & $\mathrm{x}$ \\
\hline${ }^{*}$ Zygosporium masonii S. Hughes & $\mathrm{x}$ & & $\mathrm{x}$ & & & $\mathrm{x}$ & & \\
\hline Total & 26 & 20 & 12 & 3 & 9 & 13 & 3 & 20 \\
\hline
\end{tabular}




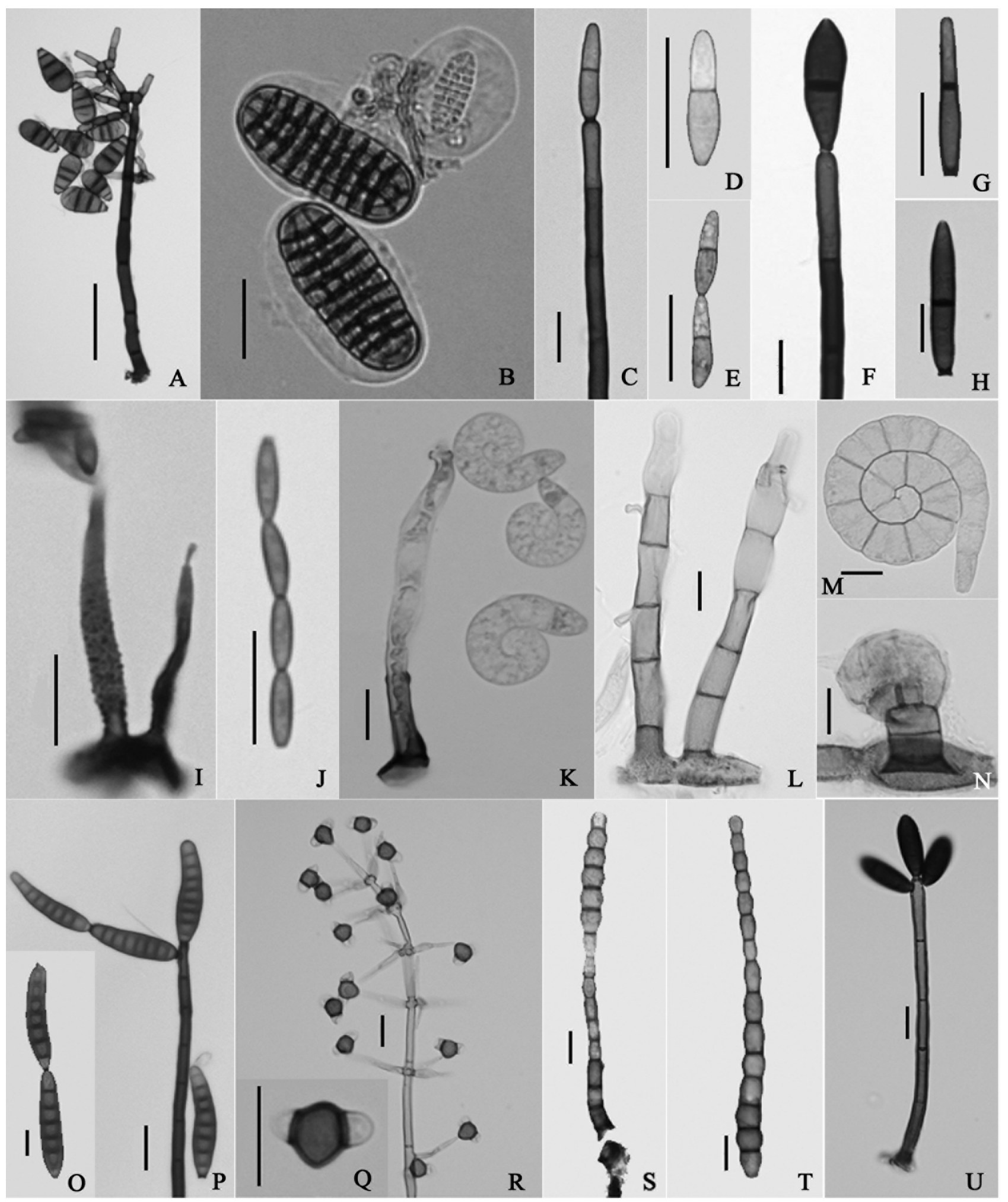

Figura 1. Conidióforos, células conidiogênicas e conídios de fungos conidiais. A. Acarocybellina arengae - aspecto geral do conidióforo com células conidiogênicas e conídios. B. Coleodictyospora micronesiaca - conídios envoltos em camada mucilaginosa. C-E. Corynesporopsis inaequiseptata. C. Ápice do conidióforo com célula conidiogênica e conídio. D. Conídio. E. Conídios em cadeia. F-H. Corynesporopsis isabelicae. F. Ápice do conidióforo com célula conidiogênica e conídio. G-H. Conídios. I-J. Gliomastix luzulae. I. Conidióforo rugoso com célula conidiogênica e conídios. J. Conídios em cadeia. K. Helicoma dennisii - conidióforo com células conidiogênicas e conídios. L-N. Helicoma viridis. L. Conidióforos com células conidiogênicas. M. Conídio. N. Esclerócio pedicelado. O-P. Helminthosporium palmigenum. O. Conídios em cadeia. P. Aspecto geral do conidióforo com células conidiogênicas e conídios. Q-R. Physalidiella elegans. Q. Conídio com células laterais sub-hialinas. R. Aspecto geral do conidióforo com células conidiogênicas e conídios. S-T. Repetophragma moniliforme. S. Aspecto geral do conidióforo com células conidiogênicas e conídio. T. Conídio constrito nos septos. U. Spadicoides macrocontinua - aspecto geral do conidióforo com células conidiogênicas e conídios. Barras $=50 \mu \mathrm{m}(\mathrm{A}) ; 20 \mu \mathrm{m}$ (B, D, E, G, P); $10 \mu \mathrm{m}$ (C, F, H, I, J, K, L, M, N, O, Q, R, S, T, U). 
Kramabeeja G.V. Rao \& K.A. Reddy para acomodar a espécie K. shrungashakha G.V. Rao \& K.A. Reddy, que apresenta uma combinação de características morfológicas semelhante a encontrada em Acarocybellina. O gênero Kramabeeja poderá ser considerado um sinônimo de Acarocybellina após estudos mais detalhados. K. shrungashakha se diferencia de A. Arengae devido a presença de conidióforos solitários ou em grupos de 2-3, medindo 250-450 × 10-15 $\mu \mathrm{m}$; células conidiogênicas cilíndricas, doliformes ou lageniformes; conídios clavados ou piriformes, com as células apicais castanho-escuro e as basais castanho-claro, 25-35 × 9,5$13,5 \mu \mathrm{m}$ (Rao et al. 1981). Enquanto na descrição original, A. Arengae possui conidióforos solitários, medindo 30-100 $\times 4-5,5 \mu \mathrm{m}$; células conidiogênicas cilíndricas e conídios obovoides, 27-37 × 12-16 $\mu \mathrm{m}$ (Matsushima 1975). Hughes (1978) registrou a maior variação de dimensão encontrada para os conidióforos (130-270 × 7-8,5 $\mu \mathrm{m}$ ) e conídios (26-41 $\times 12,5-18 \mu \mathrm{m})$ de A. Arengae. Os conidióforos e os conídios dos espécimes estudados foram maiores que os descritos por Matsushima (1975) sobre folhas mortas de Arenga engleri Becc. Apesar destas diferenças, as demais características morfológicas dos espécimes estudados estão de acordo com Matsushima (1975) e Hughes (1978).

Distribuição geográfica conhecida: Cuba (Mercado 1984), Japão (Matsushima 1975), México (Becerra et al. 2008), Peru (Matsushima 1993a), Venezuela (Castañeda et al. 2003) e Nova Zelândia (Hughes 1978).

2. Coleodictyospora micronesiaca (Matsush.) Matsush. [como 'micronesica'], Matsush. Mycol. Mem. 5: 8. 1987. $\equiv$ Berkleasmium micronesiacum Matsush. [as 'micronesicum'], Matsush. Mycol. Mem. 2: 2. 1981.

Fig. 1B

Colônias em esporodóquios, castanhas. Conidióforos micronemáticos, mononemáticos, lisos, hialinos a castanho-claros. Conídios solitários, muriformes com 8-10 septos transversais, cilíndrico-elipsoides, simples, lisos, envoltos por uma massa mucilaginosa hialina, castanho-claros, 35-47 $\times 16-20 \mu \mathrm{m}$, às vezes constritos na região central.

Material selecionado: BRASIL. Pará: Belém, Ilha do Combu, 20/VIII/2008, sobre bainhas, C.C. Castro 1- 2 (MG195837); ibid, 03/IV/2009, sobre raízes, C.C. Castro 73 (MG199674).

Comentários: Coleodictyospora Charles é composto por duas espécies (C. cubensis Charles e C. micronesiaca), que se diferenciam pelo tipo de conidióforos. Coleodictyospora cubensis apresenta conidióforos macronemáticos (70-85 $\times 3,5-5 \mu \mathrm{m}$ ), enquanto que em C. micronesiaca, segundo ilustrados e descritos por Matsushima (1987) são ausentes ou reduzidos a uma célula conidiogênica $(2-8 \times 3-4 \mu \mathrm{m})$, ou curtos ou micronemáticos, mas não oferece as dimensões (Matsushima 1989) Na descrição original Matsushima (1981) descreveu os conídios como cilíndricos, posteriormente os descreve como cilíndrico-elipsoides (Matsushima, 1987), com dimensões que variaram entre 30-40 × 13-16 $\mu \mathrm{m}$, portanto menores do que os espécimes examinados (35-47 × 16-20 $\mu \mathrm{m})$. Porém, as dimensões dos conídios dos exemplares encontrados por Hernández \& Mena (1995) sobre pecíolo de Roystonea regia (Kunth) O.F. Cook foram semelhantes as do material estudado, confirmando a identificação desta espécie.

Distribuição geográfica conhecida: Austrália, Ilhas Seychelles e Malásia (Taylor \& Hyde 2003), Brunei (Fryar et al. 2004), Cuba (Hernández \& Mena 1995), Estados Federados da Micronésia (Matsushima 1981), Estados Unidos (Delgado 2009), Hong Kong (Ho et al. 2002), México (Becerra et al. 2008), Peru (Matsushima 1993a), Tailândia (Pinnoi et al. 2006) e Taiwan (Matsushima 1987).

3. Corynesporopsis inaequiseptata Matsush., Matsush. Mycol. Mem. 7: 48. 1993.

Figs. 1C-E

Colônias efusas em substrato natural. Conidióforos macronemáticos, mononemáticos, solitários, cilíndricos, eretos, retos, simples, septados, lisos, castanho-escuros, levemente mais claros no ápice, 150-170 × 4-6 $\mu \mathrm{m}$. Células conidiogênicas monotréticas, terminais, integradas, determinadas. Conídios catenados, com um septo fino acima da parte média, obclavados, lisos, secos, parede celular fina, com a célula basal castanho-clara e a célula apical mais clara, 18-22 × 4-5 $\mu \mathrm{m}$.

Material examinado: BRASIL. Pará: Belém, Ilha do Combu, 20/VIII/2008, sobre cachos, C.C. Castro 6 (MG195842); ibid, 20/VIII/2008, sobre raques, C.C. Castro 3 (MG195838).

Comentários: Corynesporopsis foi estabelecido por Kirk (1981) para acomodar C. quercicola (Borowska) P.M. Kirk, previamente descrita como Corynespora quercicola Borowska. Atualmete o gênero é composto por 10 espécies (Castañeda $e t$ al. 2010). Das quatro espécies de Corynesporopsis P.M. Kirk que possuem conídios uni-septados, C. isabelicae Hol.-Jech. é o que mais se assemelha a $C$. inaequiseptata. Porém, estas espécies se diferenciam facilmente pela morfologia dos conídios. C orynesporopsis isabelicae apresenta conídios fusiforme-alongados ou navicular-alongados, com parede celular espessa, castanhos, com 1 septo escuro e espesso, base cicatrizada, medindo 27 3,5 × 4-6,4 um (Holubová-Jechová 1987), enquanto que $C$. inaequiseptata possui conídios obclavados, com parede celular fina, a célula basal castanho-clara e a célula apical mais clara, medindo 17-25 × 4-5,5 $\mu \mathrm{m}$ (Matsushima 1993a). Apesar de os espécimes descritos por Matsushima (1993a) terem apresentado conidióforos $(35-140 \mu \mathrm{m})$ e conídios $(17-25 \mu \mathrm{m})$ maiores em comprimento que os do material estudado, as demais características morfológicas dos espécimes brasileiros estão de acordo com a descrição original.

Distribuição geográfica conhecida: Hong Kong (Photita et al. 2001) e Peru (Matsushima 1993a).

4. Corynesporopsis isabelicae Hol.-Jech., Česká Mykol. 41(2): 109. 1987.

Figs. 1F-H 
Colônias em substrato natural efusas. Conidióforos macrocronemáticos, mononemáticos, solitários, cilíndricos, eretos, retos, simples, septados, lisos, castanho-escuros, com paredes espessas, 51-140 $\times$ 4-5 $\mu \mathrm{m}$. Células conidiogênicas monotréticas, terminais, integradas, determinadas ou percorrentes. Conídios solitários ou em curtas cadeias, 1-septados, fusiforme-alongados ou navicular-alongados, lisos, secos, castanhos, às vezes com a célula apical mais clara, parede celular espessa, $16-31 \times 4-7 \mu \mathrm{m}$; base cicatrizada com 2-3 $\mu \mathrm{m}$ de largura, célula apical mais estreita que a célula basal.

Material examinado: BRASIL. Pará: Belém, Ilha do Combu, 11/III/2009, sobre bainhas, C.C. Castro 56 (MG199657); ibid, 20/IIIV/2008, sobre cachos, C.C. Castro 5-1 (MG195841).

Comentários: as características morfológicas dos conidióforos e conídios dos espécimes estudados estão de acordo com as descritas originalmente por Holubová-Jechová (1987). Entretanto, as medidas dos conídios da descrição original $(27-43,5 \times 4-6,4 \mu \mathrm{m})$ apresentaram maior variação em relação ao material estudado $(16-31 \times 4-7 \mu \mathrm{m})$. Matsushima (1993a) descreveu os conidióforos com 150-250 $\times 6-7 \mu \mathrm{m}$, maiores do que aqueles descritos originalmente $(50-150 \mu \mathrm{m})$

Distribuição geográfica conhecida: China (Zhuang 2001), Costa Rica (Mercado et al. 1997a), Cuba (Holubová-Jechová 1987), Hong Kong (Lu et al. 2000) e Peru (Matsushima 1993a).

5. Gliomastix luzulae (Fuckel) E.W. Mason ex S. Hughes, Can. J. Bot. 36: 769. 1958.

$\equiv$ Torula luzulae Fuckel, Jb. nassau. Ver. Naturk. 23-24: 348

(1870) [1869-70]

Figs. 1I-J

Colônias efusas em substrato natural, esverdeadas, flocosas. Conidióforos macronemáticos, mononemáticos, subulados, eretos, retos ou flexuosos, ocasionalmente bifurcados na base, 1-2-septados, verrucosos, hialinos a oliváceos, 27-40 x 2,5-4 $\mu \mathrm{m}$, ápice com 0,8-1,2 $\mu \mathrm{m}$ de largura. Células conidiogênicas monofialídicas, terminais, integradas, determinadas, lisas ou rugosas, hialinas a oliváceas. Conídios em longas cadeias ou agregados em massas mucilaginosas, asseptados, fusiformes, lisos, oliváceos, 6-8 $\times 2 \mu \mathrm{m}$, com as extremidades truncadas.

Material examinado: BRASIL. Pará: Belém, Ilha do Combu, sobre raques, 11/III/2009, C.C. Castro 47 (MG199648).

Comentários: Gliomastix foi proposto por Guéguen (1905) para acomodar G. chartarum Guég. (三 Torula chartarum Corda). Este gênero tem sido taxonomicamente relacionado com o gênero Acremonium Link., desde que Gams (1971) reduziu Gliomastix a uma das três seções que compõem Acremonium. Porém, muitos autores como Matsushima (1975, 1980, 1993), Ellis (1976) e Lechat et al. (2010) consideram Gliomastix como um gênero distinto.
Dickinson (1968) ao revisar o gênero Gliomastix descreveu os conídios de G. luzulae com dimensões que variaram de 3,9-9,3 × 1,5-2,9 $\mu \mathrm{m}$. Nos exemplares encontrados por Mercado \& Heredia (1994), sobre palmeira no México, os conídios tiveram medidas semelhantes $(6-7 \times 2 \mu \mathrm{m})$ ao material estudado, enquanto que os conidióforos foram menores $(16-23 \times 2-3 \mu \mathrm{m})$. As características morfológicas observadas dos conidióforos e conídios são típicas da espécie e foram determinantes para sua identificação, embora ocorram diferenças nas dimensões.

Distribuição geográfica conhecida: Austrália (Paulus et al. 2006), Alemanha, Bélgica, Gana, Inglaterra, Itália, Malásia, Nova Zelândia e Serra Leoa (Dickinson 1968), África Ocidental, Europa e Nova Zelândia (Ellis 1971), China (Pan et al. 2009), Egito (Mouchacca 2009), México (Mercado \& Heredia 1994), Japão (Matsushima 1975), Paquistão (Matsushima 1993b), Polônia (Mulenko et al. 2008) e Suriname (Gams 1975).

6. Helicoma dennisii M.B. Ellis, Mycol. Pap. 87: 23. 1963.

Fig. $1 \mathrm{~K}$

Colônias efusas em substrato natural. Conidióforos macronemáticos, mononemáticos, solitários, cilíndricos, eretos, retos ou flexuosos, simples, 7-14-septados, lisos, castanho-pálidos com a base castanha, 77,5-220 × 4-8 $\mu \mathrm{m}$. Células conidiogênicas monoblásticas ou poliblásticas, terminais ou intercalares, integradas, determinadas, cilíndricas, denticuladas (1-2 x 0,5-1 $\mu \mathrm{m})$, lisas. Conídios solitários, 6-8 septos obscuros, levemente enrolados em um plano, 11/4-13/4 voltas, simples, lisos, secos, sub-hialinos a amarelo-pálidos, com a célula basal levemente mais escura, não higroscópicos, 15-18 $\mu \mathrm{m}$ de diâmetro, filamento conidial com 6-8 $\mu \mathrm{m}$ de diâmetro, célula basal cônico-truncada.

Material examinado: BRASIL. Pará: Belém, Ilha do Combu, 11/III/2009, sobre raques, C.C. Castro 56 (MG199657).

Comentários: Zhao et al. (2007) ao revisarem o gênero Helicoma consideraram 45 espécies, e Gawas \& Bhat 2007) publicaram mais uma espécie (Helicoma indicum Gawas \& Bhat). Helicoma dennisii é comparável a $H$. ambiens Morgan, $H$. chlamydosporum Shearer, $H$. divaricata Hol.-Jech. e H. inflatum Linder, por apresentarem conídios helicoidais com poucas voltas e filamento largo, esta espécie diferencia-se das citadas por apresentar conídios de 15-18 $\mu \mathrm{m}$ de diâmetro, com filamentos de 6-8 $\mu \mathrm{m}$ de diâmetro, não higroscópico, célula basal cônico-truncada e levemente mais escura que o resto do filamento, enquanto que Helicoma. ambiens produz conídios com $18-20 \mu \mathrm{m}$, célula basal abruptamente arredondada, em forma de U (Linder 1929, Pirozynski 1972); Helicoma chlamydosporum tem como principal característica a produção de clamidósporos que o diferencia das demais espécies (Shearer 1987); Helicoma divaricata produz conídios com 11,5-16 $\mu \mathrm{m}$ de diâmetro, com filamento de 4,5-6,5 $\mu \mathrm{m}$ diam.; Helicoma inflatum distingue-se por produzir célula apical do conídio hialina 
e inflada, filamento de 4-7 $\mu \mathrm{m}$ de diâmetro (Linder 1933). Na descrição original de H. dennisii, Ellis (1963) descreveu conidióforos com até $470 \mu \mathrm{m}$ de comprimento e conídios hialinos a sub-hialinos com 9-22 $\mu \mathrm{m}$ de diâmetro. Enquanto que no material estudado as medidas dos conidióforos (até $220 \mu \mathrm{m}$ de comprimento) e dos conídios sub-hialinos a amarelo-pálidos (15-18 $\mu \mathrm{m}$ de diâmetro) foram menores, porém próximos aos conidióforos dos espécimes descritos por Matsushima (1971) das ilhas de Salomão e Papua-Nova Guiné, e os conídios com coloração semelhante aos descritos por Matsushima (1975) do Japão e Chen \& Tzean (2010) de Taiwan. Por esse motivo, apesar das diferenças entre o material estudado e a descrição original, optamos por alocar estes espécimes em $H$. dennisii.

Distribuição geográfica conhecida: África do Sul (Lee et al. 2004), China, Estados Unidos (Zhao et al. 2007), Cuba (Delgado et al. 2002), Havaí (Goos 1980), Índia (Agarwal et al. 1993), Japão (Matsushima 1975), Nova Guiné (Matsushima 1971), Rússia (Mel'nik et al. 2007), Taiwan (Chen \& Tzean 2010) e Venezuela (Ellis 1976).

7. Helicoma viridis (Corda) S. Hughes, Can. J. Bot. 36 : 772. 1958.

$\equiv$ Helicocoryne viridis Corda [como 'viride'], Icon. fung.

(Prague) 6: 9. 1854.

Figs. 1L-N

Colônias efusas em substrato natural. Conidióforos macronemáticos, mononemáticos, solitários, cilíndricos com o ápice arredondado, eretos, retos ou flexuosos, simples, 4-8-septados, lisos com a célula basal verrucosa, castanhos na base a castanho-claros no ápice, 80-115 ×7-9 $\mu \mathrm{m}$. Células conidiogênicas monoblásticas ou poliblásticas, intercalares, integradas, determinadas, cilíndricas, denticuladas, $4-5 \times$ 2-3 $\mu \mathrm{m}$. Conídios solitários, com 20-25 septos levemente constritos, $1 \frac{1}{2}-2 \frac{1}{2}$ voltas em um plano, simples, lisos, secos, amarelo-claros, não higroscópicos, 30-36 $\mu \mathrm{m}$ de diâmetro, filamento conidial com $7 \mu \mathrm{m}$ de diâmetro. Conídios secundários não observados. Esclerócios pedicelados presentes.

Material examinado: BRASIL. Pará: Belém, Ilha do Combu, 18/III/2009, sobre pecíolos, C.C. Castro 69 (MG199670).

Comentários: Helicoma viridis apresenta uma ampla distribuição, muitos sinônimos e combinações de diferentes autores (Zhao et al. 2007). Esta é uma espécie muito comum em material vegetal em decomposição e no solo. Helicoma atroseptatum Linder é a espécie do gênero mais próxima de $H$. viridis por apresentarem a célula basal do conídio truncada, sem cicatriz conidial, conídios aderidos centralmente à célula conidiogênica e conídios secundários presentes. Helicoma. atroseptatum diferencia-se pelos conidióforos mais compridos $(150-310 \mu \mathrm{m})$, filamento mais espesso (8-11 $\mu \mathrm{m})$ e maior número de septos (10-16), enquanto que $H$. viridis apresenta conidióforos menores (75-175 $\mu \mathrm{m})$, filamento menos espesso (4-7 $\mu \mathrm{m})$ e de 5-11 septos (Zhao et al. 2007). Helicoma viridis também se as- semelha a Helicosporium pannosum (Berk. \& M.A. Curtis) R.T. Moore por apresentarem conidióforo macronematosos, eretos e robustos e células conidiogênicas dentadas (Zhao et al. 2007), porém $H$. pannosum apresenta conídios higroscópicos, 11/2-5 voltas, 12-60 septos e filamentos com até 13,5 $\mu \mathrm{m}$ de diâmetro (Pirozynski 1972), enquanto que $H$.

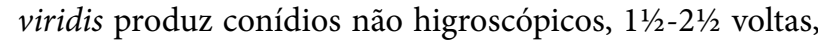
20-25 septos e filamentos com até $7 \mu \mathrm{m}$ de diâmetro Linder (1929). Os espécimes estudados de H. viridis apresentaram conídios com maior variação no diâmetro e número de septos quando comparados aos espécimes descritos por Linder (1929) e Zhao et al. (2007). Conídios secundários foram descritos por Goos (1986, 1989), mas não foram observados no material estudado. Conídios levemente constritos nos septos, semelhantes aos do material estudado, foram ilustrados por Matsushima (1975). O gênero Helicoma Corda caracteristicamente apresenta conidióforos lisos (Zhao et al. 2007), porém no presente trabalho foram observados alguns conídios com a base rugosa, caráter este não relatado anteriormente.

Distribuição geográfica conhecida: cosmopolita (Goos 1989) incluindo Alemanha e Grã-Bretanha (Saccardo 1886), China (Zhao et al. 2007), Estados Unidos (Linder 1929), Jamaica (Goos 1975), Japão (Matsushima 1975) e Tanzânia (Pirozynski 1972).

8. Helminthosporium palmigenum Matsush., Microfungi of the Solomon Islands and Papua-New Guinea (Osaka): 30. 1971.

Figs. 1O-P

Colônias efusas em substrato natural. Conidióforos macronemáticos, mononemáticos, solitários, cilíndricos, eretos, retos ou flexuosos, simples, 7-10 septados, lisos, castanhos, castanho-claros no ápice, 132,5-195 × 5-6 $\mu \mathrm{m}$. Células conidiogênicas politréticas, terminais e intercalares, integradas, determinasdas. Conídios solitários, ocasionalmente em pequenas cadeias, 6-10 pseudoseptos transversais, obclavados ou cilíndricos, retos ou levemente curvados, simples, lisos, secos, castanhos com a célula apical castanho-clara, 38-53 $\times 8-11 \mu \mathrm{m}$, base geralmente truncada e cicatrizada com 3-4 $\mu \mathrm{m}$ de largura.

Material examinado: BRASIL. Pará: Belém, Ilha do Combu, 03/IV/2009, sobre pecíolos, C.C. Castro 41 (MG199642); ibid, 20/VIII/2008, sobre raques, C.C. Castro 9- 2 (MG185845).

Comentários: o gênero Helminthosporium foi descrito por Link em 1809 originalmente sob o nome Helmisporium (Lizon \& Samuels 1997). Siboe et al. (1999) consideram 27 espécies compondo Helminthosporium. Posteriormente 14 espécies foram incluídas por Zhang et al. (2004, 2007, 2010), Shirouzu \& Harada (2008) e Zhang \& Zhang (2009). Matsushima (1971) descreveu H. palmigenum com os conidióforos menores em comprimento $(70-180 \mu \mathrm{m})$, porém mais largos $(7-10 \mu \mathrm{m})$ e conídios menores $(27-47 \times 6,5-9$ $\mu \mathrm{m})$ em relação aos do material examinado. Apesar das 
diferenças na mensuração dos conidióforos e conídios o material estudado apresentou características morfológicas semelhantes àquelas descritas originalmente, as quais não deixam dúvida sobre a sua identidade.

Distribuição geográfica conhecida: Austrália (Matsushima 1989), Cuba (Mercado et al. 1997b), Ilhas Salomão (Matsushima 1971), México (Becerra et al. 2007), Nova Zelândia (Hughes 1978), Peru (Matsushima 1993a), Taiwan (Matsushima 1980), Venezuela (Castañeda et al. 2003).

9. Physalidiella elegans (Mosca) Rulamort, Bull. Soc. bot. Centre-Ouest, Nouv. sér. 21: 512. 1990.

$\equiv$ Physalidium elegans Luppi Mosca, Allionia 11: 78. 1965. Figs. 1Q-R

Colônias efusas em substrato natural. Conidióforos macronemáticos, monomemáticos, solitários, subulados, eretos, retos ou flexuosos, ramificados, septados, lisos, castanho-claros, 137,5-225 $\times$ 5-8 $\mu \mathrm{m}$, ramos verticilados com 12-20 × 2-3 $\mu \mathrm{m}$. Células conidiogênicas monoblásticas, integradas no ápice dos ramos, determinadas, às vezes percorrentes, lisas. Conídios solitários, secos, lisos, compostos por 3 células; uma célula central obovoide ou elipsoide, castanha, 6-7 × 5-6 $\mu \mathrm{m}$ e duas células laterais hemiesféricas, sub-hialinas, $2-3 \times 3-4 \mu \mathrm{m}$.

Material examinado: BRASIL. Pará: Belém, Ilha do Combu, 20/VIII/2008, sobre cachos, C.C. Castro 6 (MG195842); ibid, sobre espatas, 03/IV/2009, C.C. Castro 53 (MG199654).

Comentários: o gênero Physalidiella Rulamort possui duas espécies: P. elegans e P. matsushimae (R.F. Castañeda $\&$ W.B. Kendr.) M. Morelet. Estas diferem entre si, principalmente, quanto à morfologia dos conídios. Physalidiella elegans produz conídios com a célula central obovoide ou elipsoide e as laterais hemiesféricas ou subesféricas (Ellis 1971), enquanto P. matsushimae apresenta conídios com a célula central fusiforme-ventricosa ou bicônica ápice frequentemente obtuso, base levemente mamiliforme e células laterais obturbinadas (Castañeda \& Kendrick 1991). Nos espécimes descritos por Ellis (1971) os conidióforos medem até $600 \mu \mathrm{m}$ de comprimento e a célula central dos conídios 7-11 $\times 6-7 \mu \mathrm{m}$, portanto maiores que nos espécimes estudados. Entretanto, as características morfológicas dos conidióforos e conídios do material estudado estão de acordo com Ellis (1971) e Arias et al. (2010).

Distribuição geográfica conhecida: Austrália (Parungao et al. 2002), Hong Kong (Zhuang 2001), Itália (Ellis 1971), México (Arias et al. 2010), Peru (Matsushima 1993a), Malásia (Matsushima 1996), Tailândia (Somrithipol et al. 2002) e Tanzânia (Pirozynski 1972).

10. Repetophragma moniliforme (Matsush.) R.F. Castañeda, McKenzie \& K.D. Hyde. Mycosphere 2: 276. 2011. $\equiv$ Sporidesmium moniliforme Matsush., Matsush. Mycol. Mem. 7: 69. 1993.

Fig. 1S-T
Colônias efusas em substrato natural. Conidióforos macronemáticos, monomemáticos, solitários, cilíndricos, eretos, retos ou flexuosos, simples, 3-10-septados, lisos, castanhos, 19-70 × 5-6 $\mu \mathrm{m}$, com a célula basal tunicada. Células conidiogênicas monoblásticas, terminais, integradas, anelídicaspercorrente, com 3-4 $\mu \mathrm{m}$ de largura. Conídios solitários, septados, moniliformes com a célula basal cônico-truncada, simples, verrucosos até a região mediana com a parte superior lisa, secos, castanho-claros, 42-91 ×7-8 $\mu \mathrm{m}$, ápice com 3,5-6 $\mu \mathrm{m}$ de largura e base com 3-4 $\mu \mathrm{m}$ de largura.

Material examinado: BRASIL. Pará: Belém, Ilha do Combu, 20/VIII/2008, sobre raques, C.C. Castro 3 (MG195839).

Comentários: o gênero Repetophragma foi proposto por Subramanian (1992) para acomodar espécies de Sporidesmium Link que apresentavam células conidiogênicas anelídicas e conídios eusseptados. Atualmente o gênero apresenta 31 espécies descritas (Castañeda et al. 2011). Análises filogenéticas indicaram que R. ontariense (Matsush.) W.P.Wu e R. goidanichii (Rambelli) W.P.Wu possuem suas fases teleomóficas associadas a classe Dothideomycetes (Shenoy et al. 2006). Repetophragma filisporum (Matsush.) R.F. Castañeda, McKenzie \& K.D. Hyde é a espécie que mais se assemelha a R. moniliforme, mas diferencia-se pelos conídios estreitamente obclavados e lisos, medindo 50-300 $\times 4-5 \mu \mathrm{m}$, ápice com $2 \mu \mathrm{m}$ de largura. Na descrição original de R. moniliforme, ainda sob o nome de Sporidesmium moniliforme, Matsushima (1993a) descreveu conidióforos (9-50 × 4-5 $\mu \mathrm{m}$ ) e conídios (55-280 $\times 6,8-9,4 \mu \mathrm{m})$ com mensurações diferentes das encontras nos espécimes estudados. Células conidiogênicas anelídicas foram observadas no material estudado, mas não foram descritas por Matsushima (1993a). No entanto, Castañeda et al. (2011) relataram a presença de 1-4 anelações nos conidióforos ao proporem a inclusão desta espécie no gênero Repetophragma. Apesar de os espécimes estudados terem apresentado conídios e conidióforos com tamanhos diferentes da descrição original, as demais características morfológicas dos mesmos concordam com Matsushima (1993a) e Castañeda et al. (2011).

Distribuição geográfica conhecida: Peru (Matsushima 1993a) e Polônia (Orłowska et al. 2004).

11. Spadicoides macrocontinua Matsush., Matsush. Mycol. Mem. 7: 67. 1993.

Fig. $1 \mathrm{U}$

Colônias efusas em substrato natural. Conidióforos macronemáticos, monomemáticos, solitários, cilíndricos, eretos, retos, simples, 6-9-septados, lisos, castanhos, 96-134 $\times 4-5 \mu \mathrm{m}$; célula apical frequentemente inflada. Células conidiogênicas politréticas produzindo conídios apicais de modo blástico e conídios laterais de modo trético, integradas, percorrentes, cilíndricas, lisas, castanho-claras. Conídios solitários, 0 -septados, obovoides ou elipsoides, simples, lisos, secos, castanho-escuros, 15-18 $\times 4-5 \mu \mathrm{m}$. 
Material examinado: BRASIL. Pará: Belém, Ilha do Combu, 03/IV/2009, sobre raque, C.C. Castro 64 (MG199665).

Comentários: o gênero Spadicoides, estabelecido por Hughes (1958), apresenta ontogenia conidial similar à de Diplococcium Grove. Ambos produzem conidióforos macronemáticos, mononemáticos com células conidiogênicas politréticas, terminais ou intercalares e conídios acropleurógenos, secos, 0-5 septados (Ellis 1971, Goh \& Hyde 1996). A separação entre os dois gêneros é feita com base na catenação dos conídios. Spadicoides possui conídios solitários, enquanto Diplococcium apresenta conídios catenados (Sinclair et al 1985, Goh \& Hyde 1996). Análises moleculares sugerem que algumas espécies de Spadicoides estão filogeneticamente relacionadas com as classes Dothideomycetes e Sordariomycetes (Shenoy et al. 2010), revelando a natureza polifilética do gênero. Conforme Ma et al. (2012) atualmente são aceitas 33 espécies em Spadicoides. Spadicoides macrocontinua apresenta semelhanças com S. atra (Corda) S. Hughes e S. subsphaerica D.W. Li com relação aos conídios unicelulares. Entretanto, S. atra possui conídios oblongos, ovoides ou elipsoides, castanho-claros a castanho-escuros, com 4-6,5 $\mu \mathrm{m}$ de comprimento (Matsushima 1975); Spadicoides subsphaerica apresenta conídios subglobosos, globosos ou elipsoides, ocasionalmente com um poro claro e protuberante na base, com 3,8$4,6 \mu \mathrm{m}$ de comprimento ( $\mathrm{Li} 2010$ ). Com isso, considerando as formas e comprimentos dos conídios apresentados por essas espécies, elas se mantêm segregadas. Os espécimes descritos por Matsushima (1993a) apresentaram os conídios maiores $(13,5-22 \times 7-9 \mu \mathrm{m})$, os conidióforos mais longos (até $140 \mu \mathrm{m}$ ) e com menor número de septos (1-5 septos), quando comparados ao material estudado. Outra diferença refere-se à presença de conidióforos ocasionalmente ramificados na descrição original (Matsushima 1993a), enquanto nos espécimes brasileiro foram simples. Entretanto as demais características morfológicas do material estudado estão de acordo com Matsushima (1993a) o que possibilitou seu enquadramento em Spadicoides macrocontinua. Este provavelmente constitui o segundo registro desta espécie para o mundo.

Distribuição geográfica conhecida: Peru (Matsushima 1993a).

\section{Agradecimentos}

Ao Curso de Mestrado em Botânica Tropical (Universidade Federal Rural da Amazônia/ Museu Paraense Emílio Goeldi), pelo apoio oferecido; aos Laboratórios de Micologia da Universidade Federal do Pará e do Museu Paraense Emílio Goeldi, pela infraestrutura cedida na realização deste trabalho; ao Conselho Nacional de Desenvolvimento Científico e Tecnológico (CNPq), pela bolsa concedida ao primeiro autor; ao Dr. Luís Fernando Pascholati Gusmão pelo auxílio nas identificações e concessão de bibliografia.

\section{Referências Bibliográficas}

Agarwal, G.P.; Gupta, S. \& Pandey, A.K. 1993. Saprophytic Fungi from Jabalpur: New Additions. Journal of Economic and Taxonomic Botany 17: 79-87.

Arias, R.M.; Heredia, G. \& Mena, J.M. 2010. Adiciones al conocimiento de la diversidad de los hongos Anamorfos del bosque mesófilo de montaña del Estado de Veracruz III. Acta Botanica Mexicana 90: 19-42.

Barbosa, F.R.; Gusmão, L.F.P. \& Barbosa, F.F. 2008. Fungos anamórficos (Hyphomycetes) no Semiárido do Estado da Bahia, Brasil. Acta Botanica Brasilica 21: 26-36.

Becerra, C.I.H.; Heredia, G. \& Arias, R.M. 2007. Los hongos anamorfos saprobios del Estado de Tabasco. II. Revista Mexicana de Micología 24: 39-53,

Becerra, C.I.H.; Heredia, G.; Arias, R.M.; Mena, P.J. \& Castañeda, R.R.F. 2008. Los hongos anamorfos saprobios del Estado de Tabasco. III. Revista Mexicana de Micología 28: 25-39.

Castañeda, R.F.R. \& Kendrick, W.B. 1991. Ninety-nine conidial fungi from Cuba and three from Canada. University of Waterloo Biology 35: $1-132$.

Castañeda, R.F.R.; Iturriaga, T.; Minter, D.W.; Saikawa, M.; Vidal, G. \& Velázquez-Noa, S. 2003. Microfungi from Venezuela. A new species of Brachydesmiella, a new combination, and new records. Mycotaxon 85: 211-229.

Castañeda, R.F.R; Silvera-Simón, C.; Gené, J.; Guarro, J.; Minter, D.M.; Stadler, M.; Saikawa, M. 2010. A new species of Corynesporopsis from Portugal. Mycotaxon 114: 407- 415.

Castañeda, R.F.R; Heredia, G.; Arias, R.M.; McKenzie, E.H.C.; Hyde, K.D.; Stadler, M.; Saikawa, M.; Gené, J.; Guarro, J.; Iturriaga, T.; Minter, D.W. \& Crous, P.W. 2011. A new species and re-disposed taxa in Repetophragma. Mycosphere 2: 273-289.

Castro, C.C.; Gutiérrez, A.H.; Sotão, H.M.P. 2011. Novos registros de fungos anamorfos (hifomicetos) para o Neotrópico e América do Sul. Revista Brasileira de Botânica 34: 515-521.

Chen, J.L. \& Tzean, S.S. 2010. Hyphomycetes from Taiwan - Endophragmia and Allied Species. Taiwania 55: 37-42.

Corda, A.C.J. 1837. Icones Fungorum hucusque cognitorum. Vol. 1. CORDA, A.C.I. 1837. Published by the author. Prague, v.1.

Dalbosco, M.; El Tassa, S.O.M. \& Duarte, V. 2004. Ocorrência de podridão negra, causada por Chalara elegans, em raízes de cenoura no Rio Grande do Sul. Fitopatologia Brasileira 29: 336-336.

Delgado, G.; Mena, J.P.; Calduch, M. \& Decock, C. 2002. Hyphomycetes (hongos mitospóricos) del Área Protegida Mil Cumbres, Cuba Occidental. Cryptogamie Mycologie 23: 277-293.

Delgado, G. 2009. South Florida microfungi: Veramycella bispora, a new palmicolous anamorphic genus and species, with some new records for the continental USA. Mycotaxon 107: 357-373.

Dickinson, C.H. 1968. Gliomastix Guéguen. Mycological Papers 115: 1-24.

Ellis, M.B. 1963. Dematiaceous hyphomycetes. IV. Mycological Papers 87: $1-42$.

Ellis, M.B. 1971. Dematiaceous Hyphomycetes. Kew, Commonwealth Mycological Institute.

Ellis, M.B. 1976. More Dematiaceous Hyphomycetes. Kew, Commonwealth Mycological Institute.

Farr, M.L. 1980. A new species of Cryptophiale from Amazonas. Mycotaxon 11: 177-181.

Fryar, S.C.; Davies, J.; Booth, W.; Hodgkiss, I.J. \& Hyde, K.D. 2004. Succession of fungi on dead and live wood in brackish water in Brunei. Mycologia 96: 219-225.

Furtado, D.C.M.; Amorim, E.P.R.; Galvão, A.L.B.; Carnaúba, J.P. \& Oliveira, M.N. 2007. Ocorrência de Curvularia lunata e Curvularia eragrostidis em Tapeinochilus ananassae no estado de Alagoas. Summa Phytopathologica 33: 201-201.

Gams, W. 1975. Cephalosporium-Like Hyphomycetes: Some Tropical Species. Transaction of the British Mycological Society 64: 389-404.

Goh, T.K. \& Hyde, K.D. 1996. Spadicoides cordanoides sp.nov., a new dematiaceous hyphomycete fom submerged wood in Australia, with a taxonomic review of the genus. Mycologia 88: 1022-1031. 
Goos, R. D. 1975. Production of secondary conidia by Helicoma proliferans. Mycologia 67: 888-891.

Goos, R. D. 1980. Some Helicosporous Fungi from Hawaii. Mycologia 72: 595-610.

Goos, R. D. 1986. A Review of the Anamorph Genus Helicoma. Mycologia 78: 744-761.

Goos, R.D. 1989. On the anamorph genera Helicosporium and Drepanospora. Mycologia 81: 356-374.

Hernández, A.G. \& Mena, J.P. 1995. Nuevos registro de hifomicetos sobre Roystonea regia en Cuba. Boletín de la Sociedad Micológica de Madrid 20:15-23.

Hernández, A.G.; Monteiro, J.S. \& Sotão, H.M.P. 2009. Hifomicetos (fungos anamorfos) associados a palmeiras na Floresta Nacional de Caxiuanã, PA, Brasil. Pp. 397-405. In: Lisboa, P.L.B (Org.). Caxiuanã: Desafios para a conservação de uma floresta nacional na Amazônia. Belém, Museu Paraense Emílio Goeldi.

Ho, W.H.; Yanna; Hyde, K.D. \& Hodgkiss, I.J. 2002. Seasonality and sequential occurrence of fungi on wood submerged in Tai Po Kau Forest Stream, Hong Kong. Fungal Diversity 10: 21-43.

Holubová-Jechová, V. 1987. Studies on Hyphomycetes from Cuba VI. New and rare species with tretic and phialidic conidiogenous cells. Česká Mykologie 41: 107-114.

Hughes, S.J. 1978. New Zealand Fungi 25. Miscellaneous species. New Zealand Journal of Botany 16: 311-370.

Hyde, K.D.; Fröhlich, J. \& Taylor, J. 1997. Diversity of Ascomycetes on palms in the tropics. Pp. 141-156. In: Hyde, K.D. (Ed.). Biodiversity of Tropical Microfungi. Hong Kong, The Hong Kong University Press.

Kirk, P.M. 1981. New or interesting microfungi. II. Dematiaceous Hyphomycetes from Esher Common, Surrey. Transactions of the British Mycological Society 77: 279-297.

Kirk, P.M. 1983. New or interesting microfungi. VIII. Corynesporopsis indica sp. nov. Mycotaxon 17: 405-408.

Lechat, C.; Farr, D.F.; Hirooka, Y.; Minnis, A.M.; Rossman, A.Y. 2010. A new species of Hydropisphaera, H. bambusicola, is the sexual state of Gliomastix fusigera. Mycotaxon 111: 95-102.

Lee, S.; Mel'nik, V.;Taylor, J.E. \& Crous, P.W. 2004. Diversity of saprobic hyphomycetes on Proteaceae and Restionaceae from South Africa. Fungal Diversity 17: 91-114.

Li, D.W. 2010. Spadicoides subsphaerica sp. nov. from Connecticut. Mycotaxon 111: 257-261.

Linder, D.H. 1929. A Monograph of the Helicosporous Fungi Imperfecti. Annals of the Missouri Botanical Garden 16: 227-388.

Linder, D.H. 1933. North american hyphomycetes. I. Two new Helicosporeae and the new genera Haplochalara and Paspalomyces. Mycologia 25: 342-348.

Lu, B.; Hyde, K.D.; Ho, W.H.; Tsui, K.M.; Taylor, J.E.; Wong, K.M.; Yanna, \& Zhou, D. 2000. Checklist of Hong Kong Fungi. Hong Kong, Fungal Diversity Press.

Ma, L.G.; Ma, J.; Zhang, Y.D. \& Zhang, X.G. 2012. Spadicoides camelliae and Diplococcium livistonae, two new hyphomycetes on dead branches from Fujian Province, China. Mycoscience 53: 25-30.

Matsushima, T. 1971. Microfungi of the Solomon Islands and Papua New Guinea. Kobe, Publicado pelo autor.

Matsushima, T. 1975. Icones Microfungorum a Matsushima Lectorum. Takashi Matsushima, Kobe. Matsushima, T. 1980. Saprophytic microfungi from Taiwan. Part 1. Hyphomycetes. Matsushima Mycological Memoirs n. 1. Matsushima Fungal Collection, Kobe.

Matsushima, T. 1981. Matsushima Mycological Memoirs n. 2. Matsushima Fungal Collection, Kobe.

Matsushima, T. 1987. Matsushima Mycological Memoirs n. 5. Matsushima Fungal Collection, Kobe.

Matsushima, T. 1989. Matsushima Mycological Memoirs n. 6. Matsushima Fungal Collection, Kobe.

Matsushima, T. 1993a. Matsushima Mycological Memoirs n. 7. Matsushima Fungal Collection, Kobe.

Matsushima, T. 1993b. List of Microfungi from Pakistan soils. Pp. 43-63. In: Nakaike T. \& Malik S. (Eds.). Cryptogamic Flora of Pakistan. 2. Tokyo, National Science Museum.

Matsushima, T. 1996. Matsushima Mycological Memoirs n. 9. Matsushima Fungal Collection, Kobe.
Mel'nik, V.A.; Popov, E.S. \& Shabunin, D.A. 2007. Contributions to the studies of mycobiota in Novgorod and Pskov regions. I. Hyphomycetes. Mikologiia i Fitopatologiia 41: 515-525.

Mercado, A.S. 1984. Hifomicetes Demaciáceos de Sierra del Rosario, Cuba. La Habana, Academia de Ciencias de Cuba.

Mercado, A.S. \& Heredia, G. 1994. Hyphomycetes associated with plant litter from the State of Veracruz, Mexico. Revista Mexicana de Micología 10: $33-48$.

Mercado, A.S.; Holubová-Jechová, V. \& Mena, J.P. 1997. Hifomicetes dematiáceos de Cuba. Enteroblásticos. Torino, Museo Regionale di Scienze Naturali.

Monteiro, J.S.; Hernández, A.G. \& Sotão, H.M.P. 2010. Fungos anamorfos (hyphomycetes) da Floresta Nacional de Caxiuanã, Pará, Brasil: novos registros para o Neotrópico. Acta Botanica Brasilica 24: 871-876.

Mouchacca, J. 2009. Novel fungal taxa from the arid Middle East introduced prior to the year 1940. II- Anamorphic fungi: hyphomycetes. Cryptogamie Mycologie 30: 199-222.

Mulenko, W.; Majewski, T. \& Ruszkiewicz-Michalska, M. 2008. A Preliminary Checklist of Micromycetes in Poland. 9. Kraków, W. Szafer Institute of Botany, Polish Academy of Sciences.

Negrão, I.; Souza, J.; Macedo, L.; Mendonça, M.; Sanches, M.; Bitar, P. \& Costa, P.F. 2009. Diversidade de fungos no estipe do açaizeiro (Euterpe oleracea Mart.). Pp. 141-145. In: Jardim M.A.G. (Ed.). Diversidade biológica das áreas de proteção ambiental Ilhas do Combu e Algodoal-Maiandeua - Pará, Brasil. Belém, Museu Paraense Emílio Goeldi, Ministério da Ciência e Tecnologia, Conselho Nacional de Desenvolvimento Científico e Tecnológico.

Nechet, K.L. \& Halfeld-Vieira, B.A. 2005. Curvularia lunata var. aeria causando queima foliar em Zoysia japonica. Fitopatologia Brasileira 30: $438-438$

Orłowska, M., Lengiewicz, I. \& Suszycka, M. 2004. Hyphomycetes Developing on Water Plants and Bulrushes in Fish Ponds. Polish Journal of Environmental Studies 13: 703-707.

Pan, H.Q.; Zhang, T.Y. \& Kong, J.H. 2009. Notes on soil dematiaceous Hyphomycetes from the Yellow River source area, China. Mycosystema 28: 14-19.

Parungao, M.M.; Fryar, S.C. \& Hyde, K.D. 2002. Diversity of fungi on rainforest litter in North Queensland, Australia. Biodiversity and Conservation 11: 1185-1194.

Paulus, B.C.; Kanowski, J.; Gadek, P.A. \& Hyde, K.D. 2006. Diversity and distribution of saprobic microfungi in leaf litter of an Australian tropical rainforest. Mycological Research 110: 1441-1454.

Photita, W.; Lumyong, S.; Lumyong, P.; Ho, W.H.; McKenzie, E.H.C. \& Hyde, K.D. 2001. Fungi on Musa acuminata in Hong Kong. Fungal Diversity 6: $99-106$

Pimentel, M.S. \& Jardim, M.A.G. 2009. Morfologia das inflorescências e infrutescências do Açaí-preto (Euterpe oleracea Mart.) e das etnovariedades Espada, Branco e Tinga (Euterpe spp.). Pp. 79-88. In: Jardim M.A.G. (Ed.). Diversidade biológica das áreas de proteção ambiental Ilhas do Combu e Algodoal-Maiandeua - Pará, Brasil. Belém, Museu Paraense Emílio Goeldi, Ministério da Ciência e Tecnologia, Conselho Nacional de Desenvolvimento Científico e Tecnológico.

Pinnoi, A.; Lumyong, S.; Hyde, K.D. \& Jones, E.B.G. 2006. Biodiversity of fungi on the palm Eleiodoxa conferta in Sirindhorn peat swamp forest, Narathiwat, Thailand. Fungal Diversity 22: 205-218.

Pirozynski, K.A. 1972. Microfungi of Tanzania. I. Miscellaneous fungi on oil palm. II. New Hyphomycetes. Mycological Paper 129: 1-65.

Rodrigues, K.F. 1994. The foliar fungal endophytes of the Amazonian palm Euterpe oleracea Mart. Mycologia 86: 376-385.

Rodrigues, K.F. \& Samuels, G.J. 1992. Idriella species endophytic in palms. Mycotaxon 43: 271-276.

Rodrigues, L.M.B.; Lira, A.U.S.; Santos, F.A. \& Jardim, M.A.G. 2006. Composição florística e usos das espécies vegetais de dois ambientes de florestas de várzea. Revista Brasileira de Farmácia 87: 45-48.

Russomanno, O.M.R.; Kruppa; P.C. \& Coutinho, L.N. 2007. Doenças fúngicas em palmeiras ornamentais. Biológico 69: 9-15.

Samuels, G. \& Müller, E. 1978. Life history studies of Brazilian Ascomycetes. 2. A new species of Thaxteriella and its helicosporous anamorph. Sydowia 31: 137-141.

Shearer, C.A. 1987. Helicoma chlamydosporum, a New Hyphomycete from Submerged Wood in Panama. Mycologia 79: 468-472. 
Shenoy, B.D.; Jeewon. R,; Wu, W.P.; Bhat, D.J. \& Hyde, K.D. 2006. Ribosomal and RPB2 DNA sequence analyses suggest that Sporidesmium and morphologically similar genera are polyphyletic. Mycological Research 110: 916-928.

Shenoy, B.D.; Jeewon, R.; Wang, H.K.; Amandeep, K.; Ho, W.H.; Bhat, D.J.; Crous, P.W. \& Hyde, K.D. 2010. Sequence data reveals phylogenetic affinities of fungal anamorphs Bahusutrabeeja, Diplococcium, Natarajania, Paliphora, Polyschema, Rattania and Spadicoides. Fungal Diversity 44:161-169.

Shirouzu, T. \& Harada, Y. 2008. Lignicolous dematiaceous hyphomycetes in Japan: five new records for Japanese mycoflora, and proposals of a new name, Helminthosporium magnisporum, and a new combination, Solicorynespora foveolata. Mycosystema 23: 179-182.

Silva, S.E.L.; Souza, A.G.C. \& Berni, R.F. 2005a. O Cultivo do Açaizeiro. Comunicado Técnico 29: 1-4. Manaus, Embrapa Amazônia Ocidental.

Silva, G.S.; Alfenas, A.C.; Alfenas, R.F. \& Zauza, E.V. 2005b. Cylindrocladium pteridis em Palmeiras Ornamentais. Fitopatologia Brasileira 30: 313.

Sinclair, R.C.; Eicker, A. \& Bhat, D.J. (1985). Branching in Spadicoides. Transactions of the British Mycological Society 85: 736-738.

Somrithipol, S.; Jones, E.B.G. \& Hywel-Jones, N.L. 2002. Fungal diversity and succession on pods of Delonix regia (Leguminosae) exposed in a tropical forest in Thailand. Fungal Diversity 10: 131-139.
Subramanian, C.V. 1992. A reassessment of Sporidesmium (Hyphomycetes) and some related taxa. Proceedings of the Indian Academy of Sciences 58: 179-189.

Sutton, B.C. 1989. Notes on Deuteromycetes. II. Sydowia 41: 330-343.

Taylor, J.E. \& Hyde, K.D. 2003. Microfungi of tropical and temperate Palms. Hong Kong, Fungal Diversity Press.

Zhang, M.; Zhang, T.Y. \& Wu, W.P. 2004. Taxonomic studies of Helminthosporium from China II. Two new species in Sichuan Province. Mycoscience 49: 126-131.

Zhang, M.; Zhang, T.Y. \& Wu, W.P. 2007. Taxonomic studies of Helminthosporium from China III. Three new species in Guangdong province. Mycotaxon 99: 399-413.

Zhang, M. \& Zhang, T.Y. 2009. Taxonomic studies of Helminthosporium from China IV. Six new species and a key to Helminthosporium from China. Mycotaxon 109: 137-142.

Zhang, M.; Wu, H.Y. \& Wang, Z.Y. 2010. Taxonomic studies of Helminthosporium from China V. Two new species in Hunan and Sichuan Province. Mycotaxon 113: 95-99.

Zhao, G.Z.; Liu, X.Z. \& Wu, W.P. 2007. Helicosporous Hyphomycetes from China. Fungal Diversity 26: 313-524.

Zhuang, W.Y. 2001. Higher Fungi of Tropical China. Ithaca, Mycotaxon Ltd. 